\title{
Management of community-acquired pneumonia
}

\author{
Mohsin Ehsan • Mark L. Metersky
}

Published online: 6 November 2013

(C) Springer Science+Business Media New York 2013

\begin{abstract}
This article summarizes recent literature related to community-acquired pneumonia (CAP), which continues to represent one of the most common causes of morbidity and mortality throughout the world, especially in the elderly. The impact is even more striking if we take healthcare costs into account. Patients with CAP usually have other comorbidities which not only increase mortality in this group but also add further to its economic burden. Patients with COPD, preexisting cardiac disease, and diabetes are particularly at higher risk for developing more severe pneumonia. New diagnostic modalities, including ultrasonography, are being used more commonly in clinical practice. Procalcitonin levels can be used to identify bacterial pneumonia and to de-escalate antibiotic therapy, resulting in fewer days on antibiotics. Other biomarkers have been used in studies to stratify patients by risk. Guideline-concordant regimens including atypical coverage, and macrolides in particular, appear to provide benefit. More recent data suggest the need for careful screening of patients for cardiovascular risk factors prior to initiating macrolides and fluoroquinolones.
\end{abstract}

\footnotetext{
M. Ehsan

Saint Joseph Health Center, St. Charles, MO, USA

M. L. Metersky

Division of Pulmonary and Critical Care, University of Connecticut

School of Medicine, Farmington, CT, USA
}

M. L. Metersky ( $₫)$

Division of Pulmonary and Critical Care, University of Connecticut

Health Center, 263 Farmington Avenue, Farmington,

CT 06030-1321, USA

e-mail: Metersky@uchc.edu

M. Ehsan $(\bowtie)$

416 Johanna Place Drive, Ballwin, MO 63021, USA

e-mail: drmohsinehsan@hotmail.com
Keywords Community-acquired pneumonia . Comorbidities · Biomarkers · Procalcitonin · Copeptin . Diagnosis $\cdot$ Antibiotic $\cdot$ Diagnosis $\cdot$ Macrolides

\section{Introduction}

Community-acquired pneumonia (CAP) is one of the most common diagnoses that physicians encounter in both the outpatient and inpatient settings. CAP results in an enormous burden on the healthcare system due to its high morbidity and mortality [1]. The increased incidence of CAP in the elderly, as well as the frequency of comorbidities in this group, further increases the impact and cost [2, 3, 4•, 5]. In 2009 in the United States, 1.1 million people with pneumonia required hospital care, with a resulting mortality of more than 50,000 [5]. The mortality rate in the rest of the world is much higher, especially among children in developing countries, exceeding mortality related to other types of infections [6]. The goals of this article are to review new literature published since January 2012. In this review, we will discuss management issues in immunocompetent adults with CAP, addressing the etiology, economic burden, impact of other comorbidities, new diagnostic modalities, risk stratification tools, and treatment modalities. We have identified potentially relevant research using a Medline search. Because this review encompasses only new data since 2012, certain topics may not be addressed.

\section{Economic burden of CAP}

Community-acquired pneumonia affects virtually every age of a population, with increasing incidence in the elderly $[2,7,8]$. From a database of approximately 13 million people in the U.S., a retrospective study was conducted on 27,659 commercially insured patients with CAP. Patients from nursing homes 
and long-term care facilities were excluded. The mean age was 62.6 years, with $72 \%$ of the patients managed as outpatients and $28 \%$ requiring inpatient treatment. The majority of the patients were stratified as low risk. CAP episodes were defined using data from claims with a primary or secondary diagnosis of pneumonia. All-cause healthcare cost of an inpatient CAP episode was $\$ 27,661$, compared to $\$ 2,221$ for an outpatient episode. The cost of inpatient management remained higher among the different age groups, with increased length of stay in older and high-risk patients [4•]. Another retrospective study involved 65,804 Medicare fee-for-service patients (representing $5 \%$ of total Medicare beneficiaries) admitted to the hospital with CAP during 2007-2008. The authors estimated 1.3 million cases of CAP in the United States, and 74,000 deaths, at a cost of $\$ 13$ billion annually. The mean age was 75 years, with $85 \%$ of the patients older than 65 years of age. Sixty-one percent of the patients were managed as outpatients, at a cost of $\$ 2,394$ per episode, and $39 \%$ were treated as inpatients, at a cost of $\$ 18$, 670 per episode [5].

It should be noted that despite having attempted to exclude nursing home and long-term care facilities, these studies may have inadvertently included some patients who might not have been true CAP. Even in a younger, working-age (18-64 years) population, CAP represents a major economic burden, with both direct medical costs and loss of productivity resulting in an estimated national cost of $\$ 10.6$ billion [9•]. CAP patients with CHF, COPD, and diabetes have substantially higher healthcare costs than patients with no comorbidities [3]. This knowledge should help identify target groups for a more robust approach to preventing CAP. Infection with resistant organisms in both healthcare- and community-associated infections also results in increased costs, hospital length of stay, and mortality [10].

\section{CAP and other diseases}

There is a growing elderly population at higher risk of developing CAP. COPD is the most common comorbidity among patients admitted for CAP, while diabetes is more common among outpatients [4•]. CAP patients with $\mathrm{CHF}$, COPD, and diabetes have substantially higher healthcare costs than patients without these comorbidities [5]. The majority of patients with CAP have coexisting cardiac disease [11]. Infections such as CAP cause a surge of inflammatory mediators and prothrombogenic milieu, resulting in instability of the coronary plaques, biomechanical stress from the interplay of vascular volume and tone, and increased load from high metabolic demand [12]. In a prospective 30-day study of 1,343 inpatients and 944 outpatients with CAP, $26.7 \%$ of inpatients and $2.1 \%$ of outpatients had cardiac events, with the majority occurring within the first week, and more than half occurring within the first 24 hours. These events included new or worsening heart failure, arrhythmia, and MI, and were associated with high 30-day mortality, despite adjusting for baseline pneumonia severity index (PSI). Groups identified at high risk for cardiac complications were older patients, nursing home residents, patients with pre-existing cardiac disease, and those with high PSI scores on admission [13•].

Between 2002 and 2010, Di Yacovo and colleagues prospectively followed diabetic and non-diabetic patients admitted with CAP. Diabetics (21.4\% of the cohort) had more comorbidities and had a higher mean PSI class. Streptococcus pneumoniae $(S$. pneumoniae) was the most common etiologic agent in both groups. Advanced age, bacteremia, septic shock at admission, and Gram-negative pneumonia were identified as risks for mortality in the diabetic group, but overall case fatality was similar in both groups $(9.2 \%$ for diabetics and $7.1 \%$ for nondiabetics). Statins, angiotensin-converting-enzyme inhibitors, aspirin, and beta-blockers did not improve outcomes in diabetic patients [14•]. The risk of developing new-onset diabetes in previously undiagnosed patients presenting with CAP and hyperglycemia was $14 \%$ in the subsequent five years. It was even higher in the moderate-to-severe hyperglycemic group, providing an opportunity to identify an at-risk population [15].

Proton pump inhibitors (PPIs) are commonly prescribed medications. We often see PPIs prescribed in the hospital and then continued after discharge without appropriate indication. Gastric acid suppression results in bacterial colonization of the stomach and alters the oropharyngeal flora. A retrospective evaluation of VA patients noted a $30 \%$ increased risk of CAP in patients receiving PPI treatment. The risk was higher in patients with dementia and in those with sedative and tranquilizer usage [16•]. PPI use was more frequently associated with $S$. pneumoniae, with a twofold increased risk of CAP compared to nonusers [17].

\section{Diagnosis of CAP}

Although CAP is usually treated empirically, an accurate determination of the infecting pathogen is useful in targeting antibiotic coverage in any infection, including CAP, and this practice leads to a decrease in antibiotic resistance, eventually reducing healthcare costs [10]. Urinary antigen testing is commonly used for S. pneumoniae and Legionella pneumophila ( $L$. pneumophila). In a small prospective study, pneumococcal urine antigen was noted to have a sensitivity of $56 \%$ in CAP. While it was helpful in facilitating withdrawal of atypical antibiotic coverage, this change did not translate into cost savings, perhaps related to the small sample size. Furthermore, since macrolide therapy may be associated with improved outcomes independent of any antimicrobial affect [18], the advantages of urinary antigen testing for $S$. pneumoniae may be even less than the authors proposed [19]. In another retrospective review, when it was compared to the gold standard of blood culture, similar sensitivity was noted [20].

Extensive diagnostic testing, including urinary antigen testing, should usually be reserved for patients with severe CAP, 
when it is more likely to yield positive results and can guide changes in therapy [21]. Once blood cultures are found to be positive, it still takes 24-48 hours for identification of the pathogen using conventional phenotypic methods. In recent years, efforts have been made to identify organisms and resistance patterns from blood cultures as soon as they are positive. Hazelton and colleagues used multiplex tandem PCR (MT-PCR) for rapid identification of common Gram-positive bacteria and antibiotic-resistance genes, and compared this method to traditional testing. The assay was able to do rapid identification within 3 hours of positive blood culture, and it was able to correctly identify pathogens, matching phenotypic identification in $96.6 \%$ of the cases [22•].

Ultrasonography done at the bedside can provide rapid information, aiding the physician in clinical decision-making without exposing the patient to radiation [23]. Shah and colleagues recently used point-of-care ultrasonography to diagnose pneumonia in children and young adults in acute care settings, and after focused training for one hour, achieved sensitivity of $86 \%$ and specificity of $97 \%$ for diagnosing lung consolidation with air bronchograms. Chest radiograph was used as standard reference. There was no statistically significant difference in diagnostic accuracy between operators with varied experience [24•]. Ultrasound can be used for early diagnosis of parapneumonic effusions and incorporated into follow-up examination for CAP patients. Ultrasonographic diagnosis of CAP can be more difficult in adults due to the challenge of the larger chest wall in obese patients and edema in critically ill patients. The spleen and air in the stomach can also interfere with the production of adequate images in the left lower chest [24•]. Although chest radiography still remains the standard for diagnosis of pneumonia, new modalities like ultrasonography can assume a greater role in diagnostic algorithm in the future as they are incorporated into training curricula and are used more commonly.

\section{Biomarkers in CAP}

Biomarkers have been studied to aid with rapid diagnosis of bacterial pneumonia, in decisions about instituting antibiotic therapy and duration of therapy. C-reactive protein (CRP) and procalcitonin (PCT) have been commonly used. CRP is nonspecific and cannot differentiate between viral and bacterial pneumonias, while an elevated PCT generally identifies bacterial pneumonia. The ProREAL study team demonstrated that adherence to a PCT algorithm to initiate and deescalate antibiotic therapy resulted in a significant reduction in antibiotic days (1.51 days). Early antibiotic cessation based on PCT algorithm did not result in adverse outcomes [25••]. España and colleagues studied 344 non-severe CAP patients and found that PCT was able to differentiate bacterial from nonbacterial etiologies. The addition of PCT or CRP to the CURB-65 score enhanced its predictive capability for hospitalization. They estimated a cutoff of PCT level of $0.15 \mathrm{ng} / \mathrm{mL}$ for predicting hospitalization and typical bacterial infection; however, $18 \%$ of the hospitalized patients had PCT levels less than the estimated cutoff. The appropriateness of admission decision for these patients was not evaluated, which makes the results difficult to apply in practice. The study was also limited by its small sample size and the lack of causative etiology in $56 \%$ of the patients [26].

Menéndez and colleagues determined the activation pattern of different cytokines and biomarkers in CAP patients in an attempt to identify causative etiology based on certain patterns. Serum levels of IL-1 $\beta$, IL-6, IL-8, IL-10, TNF- $\alpha$, PCT, and CRP were determined in patients hospitalized for CAP. Efforts were made to determine the etiology, although a diagnosis was made in only 295 (43\%) of the 685 patients. S. pneumoniae was the most common causative organism and caused bacteremia in $36(7 \%)$ of the 48 patients with bacteremia. PCT and CRP were higher in patients with bacteremia, as predicted by a PCT level $\geq 0.36 \mathrm{ng} /$ $\mathrm{mL}$ (sensitivity $85 \%$, specificity $42 \%$, and negative predictive value $98 \%$ ). IL-6 levels were high in patients with known etiology, bacteremia, and severe sepsis due to Gram-positive cocci and Gram-negative bacilli. Patients with bacteremia also had high TNF $\alpha$ levels. L. pneumophila patients had higher IL-8 and TNF- $\alpha$ compared to $S$. pneumoniae. Patients who had received antibiotics prior to admission had reduced levels of PCT, IL-6, and IL-8, making these markers less reliable in guiding antibiotic therapy in this group. Different etiological agents caused different cytokine activation patterns, but the overall predictive value of the cytokine activation pattern was limited. Given the nonspecificity of the results despite the fairly large patient population, it is unlikely that the determination of cytokine activation pattern will provide diagnostic utility in the foreseeable future [27].

Biomarkers have been used to predict severity and riskstratify patients in CAP. Nowak and colleagues studied the use of N-terminal pro-B-type natriuretic peptide (NT-proBNP), mid-regional pro-atrial natriuretic peptide (MR-proANP), and B-type natriuretic peptide (BNP) for predicting mortality in patients with CAP. Pneumonia severity index (PSI) and CURB-65 were calculated at the time of presentation, with $23 \%$ of the patients classified as PSI class V. The median follow-up was 942 days. Natriuretic peptide (NP) levels were higher in patients with short-term (30 days) and long-term mortality, and there was no significant difference in the predictive capability of different NPs which was comparable to PSI scores. NT-proBNP and MR-proANP performed better than BNP in predicting long-term mortality. The predictive capability was significant even when patients with a history of congestive heart failure were excluded. PSI point value performed better than CURB-65, CRP, and leukocyte count in predicting short-term and long-term mortality. Patients were stratified accurately into low, medium, and high-risk categories by using a combination of categorical PSI point and NTproBNP levels, with long-term mortality reaching $26 \%$ in the 
low-risk and $77 \%$ in the high-risk group. The addition of NTproBNP to PSI score was able to provide better risk stratification than PSI alone. This study was limited by its single center design and small sample size [28•].

New biomarkers have been studied to determine if their use can improve risk stratification of CAP patients in conjunction with PSI, ATS/IDSA minor criteria, and CURB-65 scores. Arginine vasopressin is an activated hypothalamic hormone that plays a role in hemodynamics and osmoregulation. Copeptin is a surrogate marker for its release. In one study, ICU admission or death was more frequent in patients admitted with CAP who had high copeptin levels. High copeptin levels were also associated with clinical instability within 72 hours after admission. Copeptin performed similarly to the PSI and ATS/IDSA minor criteria and was better in predicting early instability and mortality than other biomarkers, including CRP, PCT, leukocyte count, and mid-regional proadrenomedullin (MR-proADM) [29]. In other studies, however, high MRproADM has been noted to carry a prognostic value not only in predicting mortality in patients with PSI class IV and V but also in predicting severity of illness and likelihood of complications, irrespective of the etiology [30-32].

The "risk of early admission to ICU" score (REA-ICU) is calculated within the first 3 days of admission for CAP to stratify patients for ICU admission [33]. Secondary analysis of patients from the proHOSP study showed that patients who developed early severe CAP were predicted with a sensitivity of $76 \%$ and negative predictive value of $96.7 \%$ using a ProADM value $\geq 1.8 \mathrm{nmol} / \mathrm{L}$ or REA-ICU risk classes III-IV. These 2 markers can be combined to adequately triage patients from the ED by identifying patients at risk for developing early severe CAP [34].

Overall, these data suggest that PCT cannot be used in decisions regarding hospitalization, but could be used more frequently in clinical practice to identify bacterial pneumonia and then to deescalate therapy, with the likely result of fewer antibiotic days. Cytokine activation pattern is nonspecific, with a limited predictive capability. NT-proBNP can be used to risk-stratify patients, but more data is required before incorporating the use of other biomarkers into practice.

\section{Treatment}

The most common typical pathogen in CAP is $S$. pneumoniae, while atypical pathogens include L. pneumophila, Mycoplasma pneumoniae, and Chlamydia pneumoniae. Most experts believe that initial empiric therapy should cover both typical and atypical pathogens, as the causative pathogen is generally not identified by the usual diagnostic modalities. Appropriate empiric therapy is especially important in critically ill patients, where mortality remains high.
In a Cochrane review published recently [35••], regimens for CAP in hospitalized patients using atypical monotherapy were compared to non-atypical coverage. The 28 trials included in the review enrolled a total of 5,939 patients. The primary outcome was mortality, which did not differ between the two treatment arms. The mortality rate was lower (adjusted mean $3.5 \%$ ) than previously reported, which likely resulted from enrollment of patients with less severe pneumonia, as is typical for antibiotic registration studies. Atypical treatment achieved better bacterial eradication, with a trend towards improved clinical success, although this advantage was lost when only high-quality studies were included. This meta-analysis mirrors prior reports that have not demonstrated improved outcomes among patients treated with atypical coverage (again, mostly antibiotic registration trials), in contrast to the many observational studies showing benefits of atypical coverage, especially macrolides. It remains unclear if these disparate results are due to unrecognized confounding in the observational studies or the non-representative patient population enrolled in antibiotic registration trials. Treatment with antibiotics active against atypical pathogens demonstrated clinical success in patients with $L$. pneumophila . There was no statistically significant difference in the frequency of adverse events between the two groups. [35••].

Asadi and colleagues [36 $\bullet^{\circ}$ ] prospectively studied mortality in 2,973 CAP patients in outpatient settings. Patients were enrolled in a non-randomized fashion, and antibiotic choice was at the physician's discretion. They found that the majority of patients were treated according to guidelines. Most patients had less severe pneumonia (PSI I and II). The group treated with atypical coverage according to the guidelines had a $77 \%$ relative reduction in 30-day all-cause mortality. Macrolide use was also associated with a significant reduction in mortality, with a $72 \%$ adjusted relative reduction in 30-day mortality compared to fluoroquinolones when adjusted for age, sex, and disease severity. This reduction in mortality with macrolides is possibly attributable to their immunomodulatory and antiinflammatory effect. Similar outcomes have been reported in other studies measuring the effect of guideline-compliant antibiotic treatment and outcomes [37, 38]. This beneficial effect of macrolides on mortality was not seen in a recent systematic review, which could have been the result of small numbers and relatively less severe CAP in the trials included, resulting in the lack of appreciable mortality benefit [39].

In a systematic review including 23 studies and 137,574 patients hospitalized for CAP, Asadi and colleagues [40••] studied in-hospital and 30-day mortality with the use of different antibiotic regimens. These included both randomized controlled trials and observational studies. In the primary analysis, there was a $22 \%$ relative reduction in mortality $(3.7 \%$ vs. $6.5 \%$ ) in patients treated with macrolide-based regimens compared to non-macrolide regimens. However, there was very high heterogeneity in the pooled studies. In a subgroup analysis of 16 studies including 12,624 patients, treatment with a 
macrolide-beta lactam combination versus fluoroquinolones had no effect on mortality. The 5 RCTs included in this review had younger patients with less severe pneumonia, resulting in lower mortality compared to the observational studies $(4.3 \%$ vs. $5.5 \%$ ). Because the majority of the patients in this analysis came from observational studies, unrecognized confounding by indication could have played a role in the observed results.

A recent Brazilian study evaluated the use of guidelineconcordant hospital admission and antibiotic treatment decisions [41•]. The majority of the patients met the criteria for hospitalization, and $59 \%$ received guideline-concordant therapy similar to United States guidelines. Overall 30-day mortality was $12.3 \%$ and 1-year mortality was $19.4 \%$, with the majority of deaths in older patients. Guideline-concordant admission and treatment conferred a non-significant trend towards mortality benefit in patients admitted for CAP. Hospital length of stay was reduced by 4 days in association with guideline-adherent treatment. However, this was a single-center study with a very small sample size and retrospective design.

The benefit of corticosteroids in pneumonia was evaluated in a meta-analysis of 9 trials (1,001 patients). There was no significant reduction in mortality with the use of corticosteroids in patients with pneumonia. Patients with severe pneumonia and prolonged treatment with corticosteroids were found to have a survival benefit. Patients treated with corticosteroids were noted to have more hyperglycemic events but no difference in gastrointestinal bleeding or infections [42].

IDSA/ATS guidelines for CAP suggest using antibiotics based on the risk for drug-resistant $S$. pneumoniae (DRSP). Risk factors for DRSP include exposure to antibiotics in the previous 3 months, presence of congestive heart failure, chronic obstructive pulmonary disease, asthma, chronic liver disease, chronic kidney disease, diabetes mellitus, alcohol use, presence of malignancy, asplenia, immunosuppressive conditions, and use of immunosuppressive drugs. In the presence of these risk factors, use of a respiratory fluoroquinolone or beta lactam with a macrolide was recommended. In the absence of these risk factors, monotherapy with a macrolide can be used, with doxycycline as alternative [21].

In a small single-center study, Jenkins and colleagues from Denver Health retrospectively evaluated the use of antibiotics to treat CAP based on the presence or absence of DRSP risk factors in patients presenting to the emergency department or an urgent care center. Approximately $50 \%$ of the patients had at least one risk factor for DRSP; however, the choice of antibiotics was similar irrespective of DRSP risk factor status. They found that in the majority of patients, antibiotics were not prescribed according to IDSA/ATS recommendations [43]. Despite its limitations, this study illustrates the dilemma that clinicians face every day in treating $\mathrm{CAP}$, weighing the risk of treatment failure versus development of antibiotic resistance.
Ott and colleagues [44 ${ }^{\bullet}$ assessed the burden of treatment failure in patients hospitalized for CAP. In this prospective study of 1,236 patients, an increased length of stay was associated with treatment failure defined as the need to switch to a broader-spectrum antibiotic regimen $\geq 72$ hours after initiating antibiotics because of deteriorating clinical status or radiological worsening. Treatment failure was observed in $15.9 \%$ of patients, with the majority occurring early in therapy. Treatment failure was more common in patients with severe CAP. Patients treated with moxifloxacin had a similar rate of treatment failure compared to those treated with beta-lactam plus a macrolide. In patients with less severe pneumonia, moxifloxacin use resulted in less treatment failure compared to beta-lactam alone or in combination with a macrolide. The median length of stay for moxifloxacin was one day less than treatment with other antibiotics ( 9 vs. 10 days). Treatment failure was associated with increase in length of stay from $15.4 \pm 7.3$ days versus $9.8 \pm$ 4.2 days. Patients treated with moxifloxacin were switched from intravenous to oral routes significantly earlier and more frequently ( $79 \%$ vs. $52.8 \%$ ) than those receiving beta-lactams.

One of the metrics used by the Joint Commission and Centers for Medicare \& Medicaid Services to measure hospital performance is antibiotic delivery within 6 hours of arrival for patients presenting to the ED with CAP. This can be a challenge in EDs with high volume and in patients presenting with atypical symptoms. In a recent study, patients seen by the triage nurse in the ED were divided into low, intermediate, and high acuity based on their presenting symptoms and vital signs. Moderate-acuity patients were then evaluated by the ED physicians for further work-up and interventions. This process, called physician-assisted triage, was retrospectively evaluated in patients with CAP to identify its impact on antibiotic delivery time. There was no significant difference in timing of antibiotic delivery after implementation of physician-assisted triage, even when adjusted for possible confounders. ED volume was found to be the driver of delay in antibiotic delivery [45].

The cardiac safety of antimicrobials commonly used in the treatment of CAP has recently been called into question. In an observational study of a Tennessee Medicaid cohort, Ray and colleagues [46••] evaluated short-term effects of azithromycin, amoxicillin, levofloxacin, or no antibiotics in causing increased risk of death from cardiac effects. Patients at increased risk of death not related to arrhythmogenic effect of medications were excluded. There was a statistically significant increase in the risk of cardiovascular death, in particular sudden cardiac death, with use of azithromycin compared to no antibiotic use or use of amoxicillin. This effect was time-limited, likely related to the 5-day course of azithromycin, and did not persist in days 6-10. The risk was higher in patients with underlying cardiac disease. Cardiovascular risk from the use of azithromycin was not significantly different from levofloxacin. In a more recent study using Danish national healthcare data, the risk of cardiovascular death was significantly higher in patients using azithromycin 
compared to antibiotic nonuse [47••]. On the other hand, there was no significant difference noted when compared to penicillin V. In patients with a known history of cardiac disease, the risk was higher with azithromycin compared to penicillin $\mathrm{V}$, although it did not reach statistical significance. The mortality in these studies appears to be limited to patients with known underlying cardiovascular disease, and the overall magnitude is low. Azithromycin is one of the most commonly prescribed antibiotics, with many potential advantages, and can still be prescribed safely by carefully screening for patients with any potential cardiovascular risk factors.

Fluoroquinolones - and moxifloxacin in particular - are known to prolong the QTc interval. In a Bayer-sponsored pooled analysis of Phase II-IV randomized active-controlled trials, the cardiac safety profile of moxifloxacin $400 \mathrm{mg}$ for different indications, including CAP, was compared with other antimicrobials [48]. A total of 28,144 patients were included, with half receiving moxifloxacin. The majority of the patients received oral antibiotics, while the rest received sequential therapy, transitioning from intravenous to oral antibiotics. Only a fraction of these patients had EKGs available for analysis of QTc interval data, so cardiac events were used as surrogates for torsades de pointes and QTc prolongation. In patients who had EKGs available for analysis, there was a small but predictable increase in QTc with the use of moxifloxacin (intravenous more than oral), which did not result in an increased risk of cardiac events. Even in high-risk patients (CAP requiring ICU admission, baseline prolonged QTc, women, and $\geq 65$ years old), there was no significant difference between the two groups. Some patients in the comparator group had treatment with azithromycin, making the results difficult to interpret. The study also excluded certain high-risk patients that we would commonly see in clinical practice.

\section{Conclusion}

Community-acquired pneumonia remains one of the most common diagnoses encountered by physicians. There are new modalities being studied and gradually incorporated into the current diagnostic armamentarium to support rapid identification of causative organisms and aid in clinical diagnoses. Biomarkers may be of use in decisions regarding initiating therapy and de-escalation, thereby providing the potential to reduce costs, length of hospital stay, and antibiotic resistance. New biomarkers may also have a role in risk stratification and severity prediction and thus may allow more appropriate triage. Guideline-adherent therapy providing atypical coverage, particularly macrolides, appears to confer benefits, but may be associated with a small increased risk of cardiac events in patients at high risk.

\section{Compliance with Ethics Guidelines}

Conflict of Interest Mohsin Ehsan and Mark L. Metersky declare that they have no conflicts of interest.

Human and Animal Rights and Informed Consent This article does not contain any studies with human or animal subjects performed by the authors.

\section{References}

Papers of particular interest, published recently, have been highlighted as:

- Of importance

•- Of major importance

1. File Jr TM, Marrie TJ. Burden of community-acquired pneumonia in North American adults. Postgrad Med. 2010;122(2):130-41.

2. Kaplan V, Angus DC, Griffin MF. Hospitalized community-acquired pneumonia in the elderly: age- and sex-related patterns of care and outcome in the United States. Am J Respir Crit Care Med. 2002;165(6):766-72.

3. Polsky D, Bonafede M, Suaya JA. Comorbidities as a driver of the excess costs of community-acquired pneumonia in U.S. commercially-insured working age adults. BMC Health Serv Res. 2012;12:379.

4. - Sato R, Gomez Rey G, Nelson S, Pinsky B. Community-acquired pneumonia episode costs by age and risk in commercially insured US adults aged $\geq 50$ years. Appl Health Econ Health Policy. 2013;11(3): 251-8. Cost to treat community acquired pneumonia is high in hospitalized patients irrespective of their age and underlying comorbidities.

5. Yu H, Rubin J, Dunning S. Clinical and economic burden of community-acquired pneumonia in the Medicare fee-for-service population. J Am Geriatr Soc. 2012;60(11):2137-43.

6. Centers for Disease Control and Prevention. National Center for Immunization and Respiratory Diseases and National Center for Emerging and Zoonotic Infectious Diseases. CDC.gov, Nov, 2012

7. Marrie TJ, Shariatzadeh MR. Community-acquired pneumonia requiring admission to an intensive care unit: a descriptive study. Medicine (Baltimore). 2007;86:103-11.

8. Restrepo MI, Mortensen EM, Velez JA, et al. A comparative study of community-acquired pneumonia patients admitted to the ward and the ICU. Chest. 2008;133:610-7.

9. • Bonafede MM, Suaya JA, Wilson KL. Incidence and cost of CAP in a large working-age population. Am J Manage Care. 2012;18(7): 380-7. CAP frequently occurs and is responsible for healthcare and productivity costs even in working age population.

10. Neidell MJ, Cohen B, Furuya Y. Costs of healthcare- and community-associated infections with antimicrobial-resistant versus antimicrobial-susceptible organisms. Clin Infect Dis. 2012;55(6): 807-15.

11. Fry AM, Shay DK, Holman RC, Curns AT. Trends in hospitalizations for pneumonia among persons aged 65 years or older in the United States, 1988-2002. JAMA. 2005;294:2712-9.

12. Corrales-Medina VF, Madjid M, Musher DM. Role of acute infection in triggering acute coronary syndromes. Lancet Infect Dis. 2010;10(2):83-92.

13. - Corrales-Medina VF, Musher DM, Wells GA. Cardiac complications in patients with community-acquired pneumonia: incidence, timing, risk factors, and association with short-term mortality. Circulation. 2012;125(6):773-81. In this prospective trial, patients with $C A P$ were noted to have cardiac complications within the first week with more than half of these events occurring in the first 24 hours. 
These events were associated with increased short term mortality in CAP patients.

14. - Di Yacovo S, Garcia-Vidal C, Viasus D. Clinical features, etiology, and outcomes of community-acquired pneumonia in patients with diabetes mellitus. Medicine (Baltimore). 2013;92(1):42-50. In this prospective observational analysis, patients with diabetes presenting with CAP had increased metabolic complications. Advanced age, bacteremia, septic shock and gram negative pneumonia were identified as risk factors for mortality.

15. MacIntyre EJ, Majumdar SR, Gamble JM. Stress hyperglycemia and newly diagnosed diabetes in 2124 patients hospitalized with pneumonia. Am J Med. 2012;125(10):1036.e17-23.

16. • Hermos JA, Young MM, Fonda JR. Risk of community-acquired pneumonia in veteran patients to whom proton pump inhibitors were dispensed. Clin Infect Dis. 2012;54(1):33-42. In this observational study, recent initiation of PPI with high doses were identified as greatest risk for developing CAP. Patients with dementia and on sedative therapy were particularly at increased risk.

17. de Jager CP, Wever PC, Gemen EF. Proton pump inhibitor therapy predisposes to community-acquired Streptococcus pneumoniae pneumonia. Aliment Pharmacol Ther. 2012;36(10):941-9.

18. Metersky ML, Ma A, Houck PM, et al. Antibiotics for bacteremic pneumonia: improved outcomes with macrolides but not fluoroquinolones. Chest. 2007;131(2):466e73.

19. Piso RJ, Iven-Koller D, Koller MT. The routine use of the urinary pneumococcal antigen test in hospitalized patients with community acquired pneumonia has limited impact for adjustment of antibiotic treatment. Swiss Med Wkly. 2012;142:w13679.

20. Selickman J, Paxos M, File Jr TM. Performance measure of urinary antigen in patients with Streptococcus pneumoniae bacteremia. Diagn Microbiol Infect Dis. 2010;67(2):129-33.

21. Mandell L, Wunderink R, Anzueto A. Infectious Diseases Society of America/American Thoracic Society consensus guidelines on the management of community-acquired pneumonia in adults. Clin Infect Dis. 2007;44 Suppl 2:S27-72.

22. - Hazelton B, Thomas L, Unver T. Rapid identification of Grampositive pathogens and their resistance genes from positive blood culture broth using a multiplex tandem RT-PCR assay. J Med Microbiol. 2013;62(Pt2):223-31. MT-PCR was able to identify Gram positive pathogens and their antibiotic resistance markers rapidly and with reliable diagnostic accuracy when compared to traditional phenotype testing after positive blood cultures.

23. Moore CL, Copel JA. Point-of-care ultrasonography. N Engl J Med. 2011;364(8):749-57.

24. - Shah VP, Tunik MG, Tsung JW. Prospective evaluation of point-ofcare ultrasonography for the diagnosis of pneumonia in children and young adults. JAMA Pediatr. 2013;167(2):119-25. Clinicians with varied experience were able to accurately identify pneumonia using point-of care ultrasonography when compared to chest radiograph.

25. - Albrich WC, Dusemund F, Bucher B, et al. Effectiveness and safety of procalcitonin-guided antibiotic therapy in lower respiratory tract infections in "real life": an international, multicenter poststudy survey (ProREAL). Arch Intern Med. 2012;172(9):715-22. In this observational study compliance with PCT algorithm resulted in safely and efficiently reducing antibiotic therapy with no added increase in risk of mortality or adverse events.

26. España PP, Capelastegui A, Bilbao A, et al. Population study of pneumonia (PSoP) group: utility of two biomarkers for directing care among patients with non-severe community-acquired pneumonia. Eur J Clin Microbiol Infect Dis. 2012;31(12):3397-405.

27. Menéndez R, Sahuquillo-Arce JM, Reyes S, et al. Cytokine activation patterns and biomarkers are influenced by microorganisms in community-acquired pneumonia. Chest. 2012;141(6):1537-45.

28. - Nowak A, Breidthardt T, Christ-Crain M, et al. Direct comparison of three natriuretic peptides for prediction of short- and long-term mortality in patients with community-acquired pneumonia. Chest.
2012;141(4):974-82. Natriuretic peptides were able to predict short and long-term mortality in CAP patients with comparable diagnostic accuracy to Pneumonia Severity Index (PSI).

29. Kolditz M, Halank M, Schulte-Hubbert B, et al. Copeptin predicts clinical deterioration and persistent instability in communityacquired pneumonia. Respir Med. 2012;106(9):1320-8.

30. Courtais C, Kuster N, Dupuy AM, et al. Proadrenomedullin, a useful tool for risk stratification in high pneumonia severity index score community acquired pneumonia. Am J Emerg Med. 2013;31(1): 215-21.

31. Bello S, Lasierra AB, Mincholé E, et al. Prognostic power of proadrenomedullin in community-acquired pneumonia is independent of aetiology. Eur Respir J. 2012;39(5):1144-55.

32. Suberviola B, Castellanos-Ortega A, Llorca J, et al. Prognostic value of proadrenomedullin in severe sepsis and septic shock patients with community-acquired pneumonia. Swiss Med Wkly. 2012;142:w13542.

33. Renaud B, Labarère J, Coma E, et al. Risk stratification of early admission to the intensive care unit of patients with no major criteria of severe community-acquired pneumonia: development of an international prediction rule. Crit Care. 2009;13(2):R54.

34. Renaud B, Schuetz P, Claessens YE, et al. Proadrenomedullin improves risk of early admission to ICU score for predicting early severe community-acquired pneumonia. Chest. 2012;142(6):1447-54.

35. •• Eliakim-Raz N, Robenshtok E, Shefet D. Empiric antibiotic coverage of atypical pathogens for community-acquired pneumonia in hospitalized adults. Cochrane Database Syst Rev. 2012;9, CD004418. In this Cochrane review, CAP regimens with and without atypical coverage were compared. Atypical coverage in hospitalized patients with CAP did not confer survival benefit.

36. •• Asadi L, Eurich DT, Gamble JM, et al. Guideline adherence and macrolides reduced mortality in outpatients with pneumonia. Respir Med. 2012;106(3):451-8. In this prospective, population based cohort based study of patients treated for CAP as outpatient. Guideline concordant antibiotic treatment and use of macrolides were associated with reduction in mortality.

37. McCabe C, Kirchner C, Zhang H, et al. Guideline concordant therapy and reduced mortality and length of stay in adults with communityacquired pneumonia: playing by the rules. Arch Intern Med. 2009;169(16):1525e31.

38. Arnold FW, LaJoie AS, Brock GN, et al. Improving outcomes in elderly patients with community-acquired pneumonia by adhering to national guidelines: Community-Acquired Pneumonia Organization International Cohort Study Results. Arch Intern Med. 2009;169(16): $1515 \mathrm{e} 24$.

39. Bjerre LM, Verheij TJ, Kochen MM. Antibiotics for community acquired pneumonia in adult outpatients. Cochrane Database Syst Rev. 2009;4, CD002109.

40. •• Asadi L, Sligl WI, Eurich DT, et al. Macrolide-based regimens and mortality in hospitalized patients with community-acquired pneumonia: a systematic review and meta-analysis. Clin Infect Dis. 2012;55(3):371-80. In this systematic review of hospitalized patients with CAP, macrolide-based regimens were associated with significant reduction in mortality. In sub-group analysis of patients only including the RCT's, this mortality benefit was lost because these patients were younger and had less severe pneumonia.

41. - Silveira CD, Ferreira CS, Corrêa Rde A. Adherence to guidelines and its impact on outcomes in patients hospitalized with communityacquired pneumonia at a university hospital. J Bras Pneumol. 2012;38(2):148-57. In this retrospective study, patients with CAP had favorable outcome with guideline adherent treatment and admission.

42. Nie W, Zhang Y, Cheng J, et al. Corticosteroids in the treatment of community-acquired pneumonia in adults: a meta-analysis. PLoS One. 2012;7(10):e47926.

43. Jenkins TC, Sakai J, Knepper BC, et al. Factors for drug-resistant streptococcus pneumoniae and antibiotic prescribing practices in 
outpatient community-acquired pneumonia. Acad Emerg Med. 2012;19(6):703-6.

44. - Ott SR, Hauptmeier BM, Erne C, et al. Treatment failure in pneumonia: impact of antibiotic treatment and cost analysis. Eur Respir J. 2012;39(3): 611-8. In this prospective, multicenter study of hospitalized patients with $C A P$, treatment failure was associated with increased costs and length of hospital stay. Initial treatment with broad spectrum antibiotics including atypical coverage was associated with less treatment failure.

45. Capp R, Soremekun OA, Biddinger PD, et al. Impact of physicianassisted triage on timing of antibiotic delivery in patients admitted to the hospital with community-acquired pneumonia (CAP). J Emerg Med. 2012;43(3):502-8.

46. •• Ray WA, Murray KT, Hall K, Arbogast PG, Stein CM. Azithromycin and the risk of cardiovascular death. N Engl J Med. 2012;366:1881-90.
In this study patients treated with azithromycin had a small but significantly increased risk of cardiovascular death compared to amoxicillin, ciprofloxacin or antibiotic non-use. The risk was more pronounced in patients with known cardiovascular disease.

47. •• Svanström H, Pasternak B, Hviid A. Use of azithromycin and death from cardiovascular causes. N Engl J Med. 2013;368:1704-12. Azithromycin use was not associated with an increased risk of death from cardiovascular causes compared to penicillin $V$, however the risk was significantly increased when compared to no use of antibiotics. In young and middle-aged adults azithromycin use was not associated with increased risk of cardiovascular death.

48. Haverkamp W, Kruesmann F, Fritsch A, van Veenhuyzen D, Arvis P. Update on the cardiac safety of moxifloxacin. Curr Drug Saf. 2012;7(2):149-63. 